\section{MS2-O4 Macromolecular Crystallography at SwissFEL}

Isabelle Martiel ${ }^{1}$, Meitian Wang ${ }^{1}$, Ezequiel Panepucci ${ }^{1}$, Claude Pradervand ${ }^{1}$, Jörg Standfuss ${ }^{1}$, Christopher J. Milne ${ }^{1}$, Gerhard Ingold $^{1}$, Bill Pedrini ${ }^{1}$, Rafael Abela ${ }^{1}$

\section{Paul Scherrer Institute, Switzerland}

email: isabelle.martiel@psi.ch

X-ray free electron lasers (XFELs) bear the promise of providing damage-free protein structures in a "diffract-before-destroy" approach. The Swiss free electron laser (SwissFEL) is currently under construction at the Paul Scherrer Institute, Switzerland. In the standard operation mode, the hard X-ray beamline will deliver 2-20 fs (rms) pulses at $100 \mathrm{~Hz}$ repetition rate, with photon energies in the range 2-12.4 $\mathrm{keV}$ and up to about 1 $\mathrm{mJ} /$ pulse. The start of operation is foreseen at the end of 2017. The hard X-ray experimental stations are conceived to offer state-of-the-art possibilities for femtosecond protein crystallography, with a variety of sample delivery methods and experimental conditions. At each station, femtosecond pump lasers will be available for time resolved studies.

The SwissFEL hard X-ray experimental station A (ESA) is designed for time resolved X-ray spectroscopy and serial femtosecond crystallography (SFX). It will cover the full photon energy range $2-12.4 \mathrm{keV}$, and the beam can be focused down to a size of $1 \mu \mathrm{m}$. The experiments are done within a chamber at pressures ranging from vacuum to ambient. The chamber can host injectors to perform SFX measurements while delivering the sample with a liquid or viscous jet [1]. Simultaneous acquisition of diffraction images and single-shot X-ray emission spectra will be possible.

The SwissFEL hard X-ray experimental station B (ESB) is designed for time-resolved X-ray diffraction studies in the field of condensed matter physics. The beam can be focused down to a size of $2 \mu \mathrm{m}$. The station also includes a general purpose table for alternative setups, one of which will be an in-house developed instrument for fixed-target protein crystallography, an important recently developed alternative to sample injection. The possible data acquisition schemes include synchrotron-like rotation measurements on a relatively small set of large crystals [2], as well as SFX on microcrystals deposited on a solid support [3] with fast scanning data acquisition, in the photon energy range 5-12.4 keV. An automatic sample changer will ensure high-throughput use of the XFEL beam. In-air and in-helium sample environments, as well as cryogenic and room-temperature conditions will be available.

[1] Kang, Y., et al. (2015). Nature, 523(7562), 561-567

[2] Cohen, A. E., et al. (2014), Proc. Natl. Acad. Sci. U. S. A., 111(48), 17122-17127.

[3] Hunter, M. S., et al, (2014). Sci. Rep., 4, 6026.

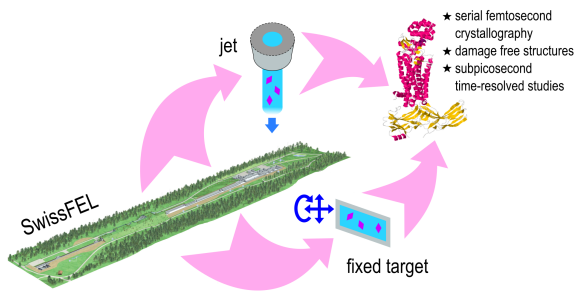

Figure 1. Sample delivery possibilities at SwissFEL. Structure of the rhodopsin-arrestin complex by femtosecond X-ray laser (PDB $4 \mathrm{ZWJ}$, ref [1]).

Keywords: Free electron laser, macromolecular crystallography, serial crystallography, femtosecond crystallography, sample delivery, fixed target, jet 